\title{
Estación de hidratación: Microarquitectura inmótica resiliente - Modelo de interacción
}

\author{
Hydration station: Inmotic Resilient Microarchitecture- Interaction Model
}

\author{
Silvia Patricia Hernández \\ FAUD. UNC Argentina \\ arqpatriciahernandez@gmail.com
}

\author{
Luciana Lanzone \\ FAUD. UNC Argentina \\ lulanzone@gmail.com
}

\author{
Raquel Landenberg \\ FAUD. UNC Argentina \\ arqcomercial.rl@gmail.com
}

\author{
José Manuel Ruiz \\ FAUD. UNC Argentina \\ interestudio@hotmail.com
}

\author{
Alejandra Rezk \\ FAUD. UNC Argentina \\ alerezk@gmail.com.ar
}

\author{
Martín Viecens \\ FAUD. UNC Argentina \\ martinviecens@yahoo.com
}

\begin{abstract}
This paper presents the research and development of a concrete micro-architecture proposal, based on a useful design for an interstitial urban space. The typology is a hydration station in the doors of an urban park, the park Sarmiento of the city of Córdoba where many gymnasts converge. The space with inmotic technology is resilient, able to mutate to be more comfortable, and is able to offer water with use control to ration in case of urban emergency. Working with domotics allows us to optimize the management of resources and therefore increase the efficiency of the systems of use and control.
\end{abstract}

Keywords: Microarchitecture; Adaptation; Resilient; Service; Interaction..

\section{Introducción}

Con esta investigación se presenta la continuación de un trabajo comenzado en el año 2016. Los autores conforman un equipo que pertenece a un programa (grupo de cuatro proyectos de investigación) aprobado ante la SECyT, Secretaría de Ciencia y Tecnología de Argentina, en el cual se trabaja en forma colaborativa e interdisciplinaria entre los distintos proyectos del programa. La propuesta de este programa se basa en la consideración de que los espacios arquitectónicos o urbanos interactúan con el ambiente, modificando, aprovechando o sufriendo las preexistencias ambientales, y en este proyecto se incluye también las posibilidades de interacción en situaciones extremas. Esta interacción puede darse en múltiples escalas. Para Monroy (2006) una adecuada calidad ambiental del hábitat construido se alcanza a partir de un conjunto de técnicas, infraestructuras y equipamientos que en conjunto definen el concepto de acondicionamiento ambiental. La calidad se produce a partir de la adaptación eficiente y sustentable de los espacios arquitectónicos y urbanos a las preexistencias, de manera progresiva desde la escala urbana a la edilicia. De tal manera que la formación del programa se orienta a generar un espacio de investigación destinado a un aspecto primordial en el desarrollo y crecimiento sustentable de la ciudad, como es el manejo de las preexistencias ambientales, su directa influencia en las condiciones de confort y calidad de vida y la necesidad de incorporarlas como condicionantes en el diseño de los edificios y los espacios urbanos.
Trabajamos en la ciudad de Córdoba, Argentina. En las condiciones actuales de la ciudad, por su escala, densidad y diversidad física y social, se hace necesaria la búsqueda y definición de estas técnicas, así como de infraestructuras y equipamientos, que adaptados con el diseño a las condiciones locales, garanticen un acondicionamiento ambiental eficiente, con el uso de energías renovables y limpias y de tecnologías e infraestructuras que avancen en la microgeneración energética, además de ofrecer un servicio al usuario de la ciudad. La problemática se puede abordar desde múltiples enfoques, todos relacionados entre sí: estudio sistemático de las preexistencias ambientales, la definición de técnicas de diseño que permitan su control y aprovechamiento o las estrategias de control ambiental a nivel urbano. En todos los casos los aportes resultantes deben confluir en la definición de normas o recomendaciones específicas. Consideramos la arquitectura con su capacidad generativa, su fuerza para crear nuevas realidades y su potencial de transformación. Es precisamente esta condición lo que la convierte en una disciplina de mutación continua y que es resiliente. Nuestra investigación con las tecnologías de vanguardia como la inmótica y los nuevos procesos de diseño permiten experimentar esta realidad de la arquitectura en prototipos digitales para situaciones concretas. (Maristany, 2016)

La arquitectura se comporta como un artefacto simbiótico que vive y se define de lo otro para crear lo propio. (Aurelli, 2012). $Y$ esa es su fuerza. El diseño se nutre de los datos y 
requerimientos de la comunidad para devolver una transformación que es un aporte a la vida.

En este proyecto se continuó con la propuesta 2016, en el mismo sitio intersticial ya analizado.

\section{Objetivo}

Nuestro objetivo es realizar una propuesta de diseño de microarquitectura urbana, con desarrollo tipológico de servicio para la ciudad de Córdoba, con tecnología inmótica, autosustentable, resiliente, que sea inclusiva, que permita al usuario interactuar con ella y en ella, que se adapte al medio, dialogando con el mismo y que también se modifique de acuerdo a requerimientos de cambio, para un espacio intersticial público urbano determinado, entendiendo su organización, y articulación con la ciudad. Trabajaremos con tecnología local, considerando los avances tecnológicos y de diseño que se están dando en el mundo y en Argentina.

\section{Metodología y desarrollo}

La metodología empleada fue la exploración sobre lo existente, en la actualidad del mundo y del propio país. Luego trabajamos con propuestas de diseño que fuimos ajustando a los objetivos. El parador se ubicó en un espacio intersticial, ya elegido en la ciudad de Córdoba.

Es sustentable, y proporcionará agua a los usuarios del parque. Realizamos una encuesta para los usuarios del parque, para determinar sus preferencias en cuanto a servicios e interacción.

a. Se elaboró un diagnóstico del estado actual del diseño y producción de microarquitectura inmótica y sustentable, considerando los ejemplos más relevantes existentes en Argentina y el mundo. Evaluándose las diferencias, calidades, protocolos, diseños, aptitudes, etc.

b. Se seleccionó un espacio intersticial para trabajar, puerta del parque Sarmiento.

c. Se realizó una Encuesta a usuarios de espacios urbanos públicos y semi-públicos, en cuanto a usos, interacción, accesibilidad, servicios requeridos etc.

d. Ensayamos de proyectos con alternativas de sistemas y de usos, Sistematizando de la información y proponiendo Hipótesis de forma, uso, tecnología y sistemas.

e. Se construyeron prototipos en modelos 3D y en maquetas virtuales.

f. Diagnóstico, Conclusiones de la situación actual. Se elaboró un diagnóstico para Argentina con propuestas de consideraciones a tener en cuenta en cada área analizada.

g. Transferencias de lo abordado:

h. En la universidad, en distintos medios,...

Se contactó ya con organismos de toma de decisiones del municipio de la ciudad, secretaría de cultura, para ofrecer el producto.

\section{Propuesta: Estación de Hidratación}

La propuesta se articula y organiza de acuerdo a los planes y normas que regulan la ciudad de Córdoba, Argentina, ya que se pretende un aporte a los organismos de gestión, para lo cual trabajamos en un proyecto construible, con tecnología local.

El trabajo está planteado en un espacio intersticial definido como el espacio que media entre dos partes de distintos objetos 0 entre dos del mismo cuerpo. La idea de intersticialidad es propia del estudio de las espacialidades urbanas contemporáneas. Antecedentes en investigaciones de espacios intersticiales permiten definir, asociación de conceptos afines tales como espacio chatarra (Koolhaas; 2002), inbetween (E2 groupe; 2003), terrain vague (Solá Morales; 1996), áreas de impunidad (Ábalos y Herreros; 1997), no lugar (Augè; 1997), entre otros, que fueron tomados como categorías antecedentes.

Se propuso una estación de hidratación en la puerta del parque Sarmiento, para la gran cantidad de deportistas que hacen gimnasia en el mismo, donde los deportistas se pueden hidratar, elongar e intercambiar o estacionar su bicicleta.

Los encuestados pidieron también bancos, y música. O sea un espacio de estar no sólo de servicio y paso.

\section{Encuesta}

Cuando trabajábamos en la propuesta de servicios para un espacio público realizamos una encuesta que arrojó los siguientes resultados.

Tenemos un universo que realiza deportes en el parque Sarmiento con una frecuencia de tres veces por semana, en un $29 \%$ y sólo los fines de semana el $38 \%$. De estos usuarios el $50 \%$ llega hasta el parque a pie. Casi el $60 \%$ considera muy útil tener equipos para elongar, que sumados a los podría ser hacen un total del $97 \%$.

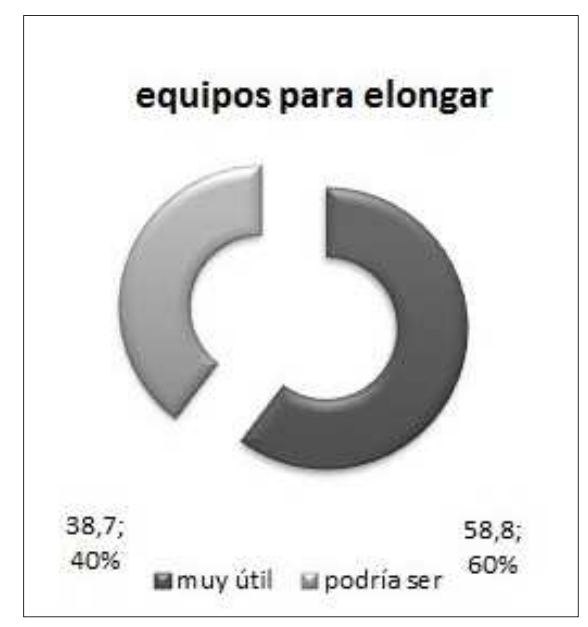

Figura 1 
El $89 \%$ de los usuarios están dispuestos a cargar su botella de agua fresca que ofrecería la estación, pero sólo el $49 \%$ pagaría por agua mineral.

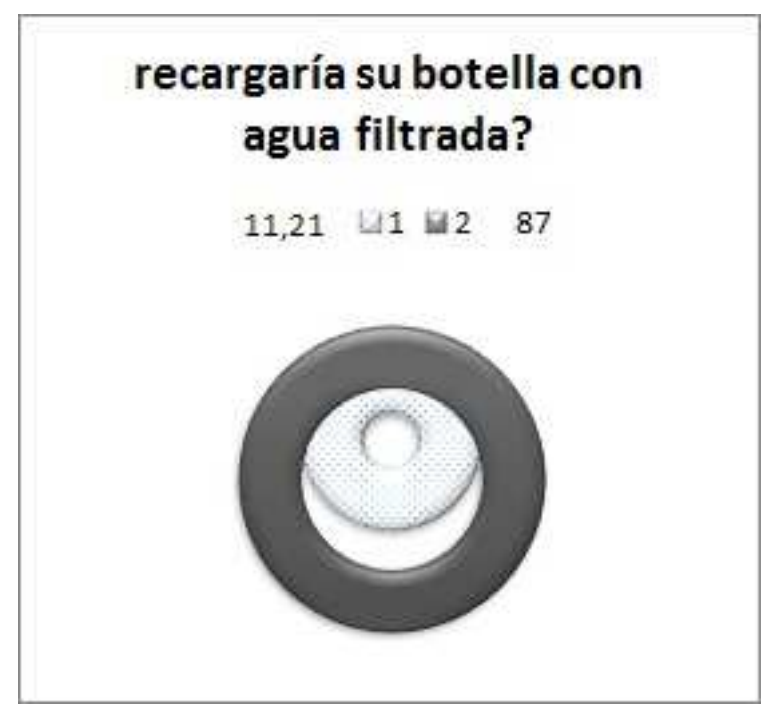

Figura 2

Lo que si aumenta considerablemente es la voluntad de los usuarios de comprar fruta fresca y llega al 79\% de usuarios. Sin embargo cuando se ofrece la venta de semillas para una alimentación saludable, el porcentaje de usuarios dispuesto a comprarlas baja al $52 \%$.

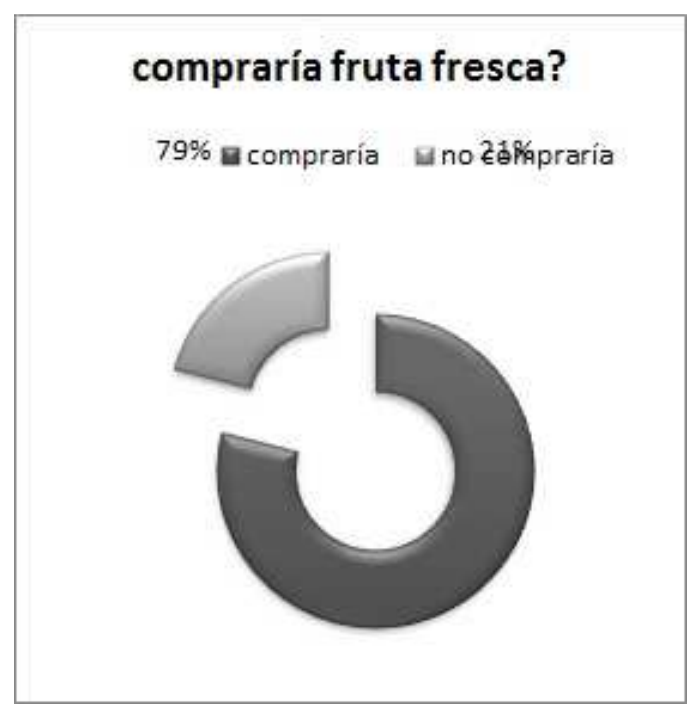

Figura 3

En la escala de valores de los encuestados ellos consideran como lo más importante resulta la recarga gratis de agua filtrada, y sigue en segundo lugar la venta de fruta fresca.

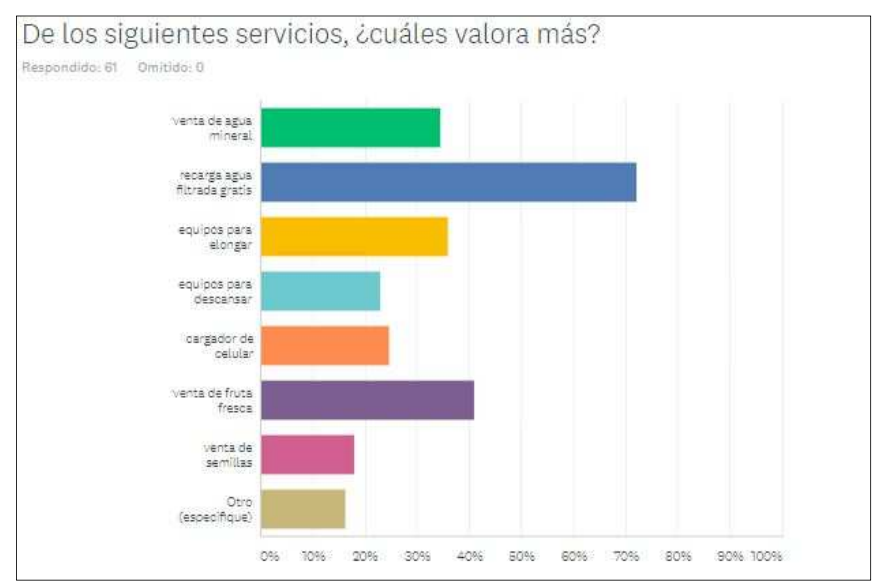

Figura 4

\section{Domótica. Inmótica}

¿Qué es la domótica? La evolución histórica indica que en un principio los sistemas aplicados en las construcciones eran de control manual. En los Estados Unidos, tras la crisis petrolera de los años 70, con el objetivo principal de generar un ahorro en el consumo, surgen las primeras automatizaciones en edificios, (Romero Morales et al, 2006). Luego, de las primeras automatizaciones con el uso de los termostatos, se comenzaron a usar los sensores para los controles de humedad, caudal de aire, etc., en los sistemas de climatización, y para los controles de intrusos, en los sistemas de alarmas.

Se la llamó domótica, que proviene de domus: casa y tica: de domotique (del francés): robótica (Enciclopedia Larousse, 1988).

La domótica surge entonces con el objetivo principal de otorgar al usuario el máximo confort y seguridad con la mayor economía y eficiencia energética.

Cuando se orienta a edificios terciarios, no viviendas, estamos ante la presencia de la inmótica, que realiza la gestión de la energía incluyendo las automatizaciones de las actividades y el trabajo. (Morales, Serrano, Lozano, 2006).

En este proyecto nos permite los movimientos del pabellón de extensión, el cerramiento, control por CCTV para el vandalismo, el control de ración de agua ante emergencia, y la generación de energía renovable controlada entre algunos sistemas.

\section{Resiliencia}

Teniendo en cuenta las definiciones de resiliencia de la física y de la sicología, se puede afirmar que en el caso del diseño de la microarquitectura inmótica, la resiliencia que proponemos se refiere a la capacidad de adaptación a distintos requerimientos, siendo la domótica la que nos posibilita más fácilmente esta adaptación. La tecnología, en nuestro caso, permite que el objeto se adapte tanto a los 
servicios y funciones que la unidad proyectada provee, como así también a su sitio y a las inclemencias climáticas. También consideramos la posibilidad de tengan la capacidad de adaptarse a catástrofes, mutando rápidamente de función o de sitio, o sea respondiendo a requerimientos de usos cotidianos $\mathrm{y} / \mathrm{o}$ de usos de emergencia.

De acuerdo con Adamo (2003), la resiliencia es un concepto utilizado inicialmente en el campo de la física y, luego, en el de la ecología, para referir la estabilidad de un sistema. Definición ésta que más tarde es reformulada y ampliada para referir la capacidad del sistema para conservar su estructura interna frente a las perturbaciones, asimilando así cambios sin tener que tergiversar lo cualitativo -es decir, sin perder las propiedades que lo caracterizan.

En ese sentido, uno de los aspectos característicos de la resiliencia social es el comportamiento del sistema debido a la estructura de sus atributos y sus interacciones entre ellos.

Tomamos la resiliencia para este proyecto dando un enfoque de un sistema complejo. Es Adger (2000) quien define la resiliencia social como la capacidad de los grupos o comunidades para hacer frente o resistir tensiones externas, los choques y perturbaciones a su infraestructura social, como resultado de los cambios sociales, políticos y ambientales. En esta definición de resiliencia social se toma en cuenta la adaptación al cambio. Así lo trabajamos en esta propuesta que se despliega, que se cierra en horarios nocturnos y que permite un control de suministro de agua en caso de emergencia sanitaria y a la vez permite elegir uno de los dos proveedores de la ciudad y tiene una tercera alternativa.

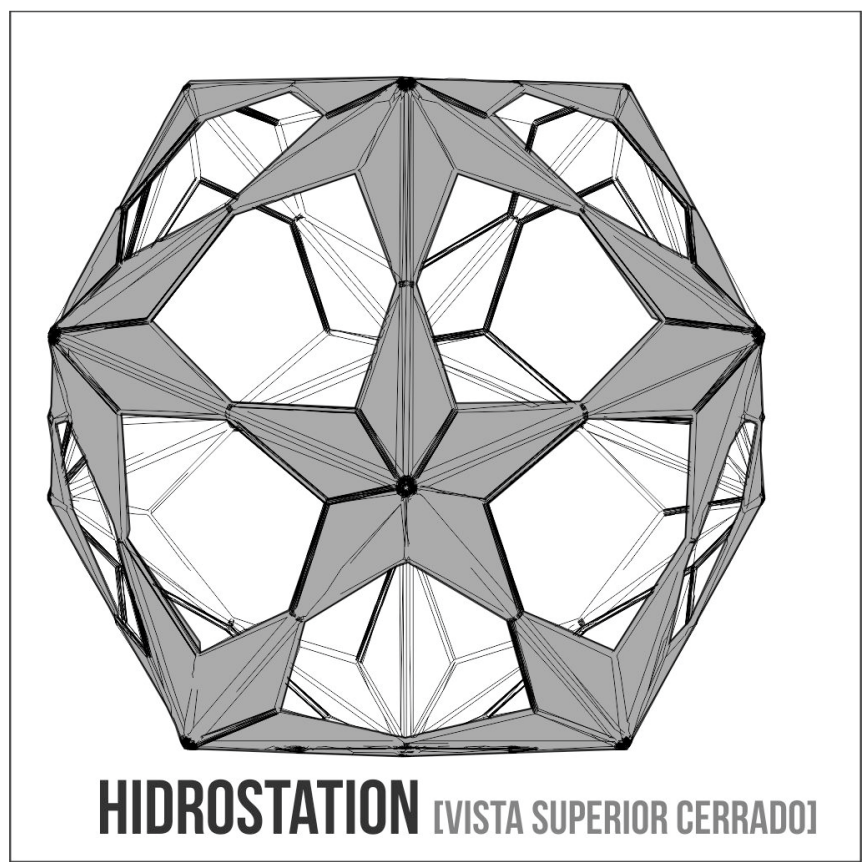

Figura 5

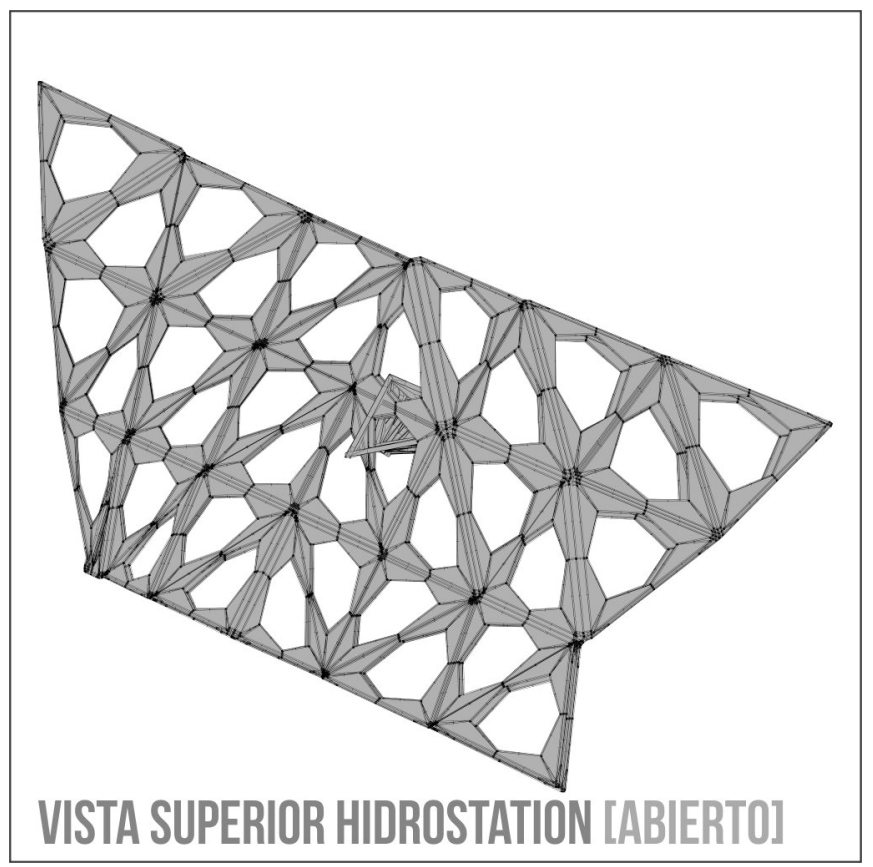

Figura 6

\section{Antecedentes de emergencias en Córdoba y la resiliencia}

Cuanto mayor es la dependencia de una comunidad respecto al reducido ámbito de aplicación de los recursos naturales, mayor será la vulnerabilidad de sus sistemas de subsistencia. Estas comunidades están sujetas tanto a la variabilidad ambiental, socio-económica así como las políticas del país.

En ese sentido, se concuerda con Adger (1999) de que la resiliencia incorpora la capacidad tanto de los sistemas sociales como los ecológicos. Ambos sistemas acusan tensiones y crisis externas. La ciudad de córdoba recibe agua de dos suministros, Dique Los Molinos y Dique San Roque y que a su vez van a dos plantas: la Planta Potabilizadora Suquía y la Planta Potabilizadora Los Molinos.

Ante lo sucedido en la ciudad de córdoba en marzo del año 2017, donde una tormenta anegó la planta de residuos peligrosos de la firma TAYM, generando derrames de afluentes turbios al canal que traslada agua a la estación potabilizadora Los Molinos. El agua que llegó al canal Los Molinos superaba los niveles permitidos de contaminación. Así lo confirmaron en horas de la mañana desde la fiscalía de Alta Gracia a cargo de Alejandro Peralta Otonello. Las muestras recogidas el día de la tormenta confirmaron que había hierro, aceite de avión y herbicida en el canal, antes de ser tratada en la planta potabilizadora. Por lo cual la zona sur de la ciudad estuvo varios días sin agua, hasta que se aseguró que el agua era nuevamente potable.(La voz del interior, 2017).

Citando a Randriamahazo (2009), que atribuye a la resiliencia social desde cuatro cualidades claves: flexibilidad, capacidad de aprender, capacidad de organización, y los activos. En un sistema flexible, es esperable que cada grupo sea robusto y 
esté vinculado a un nivel local, regional y nacional; lo que quiere decir que en un sistema flexible no puede haber un grupo débil o que falten vínculos entre las escalas.

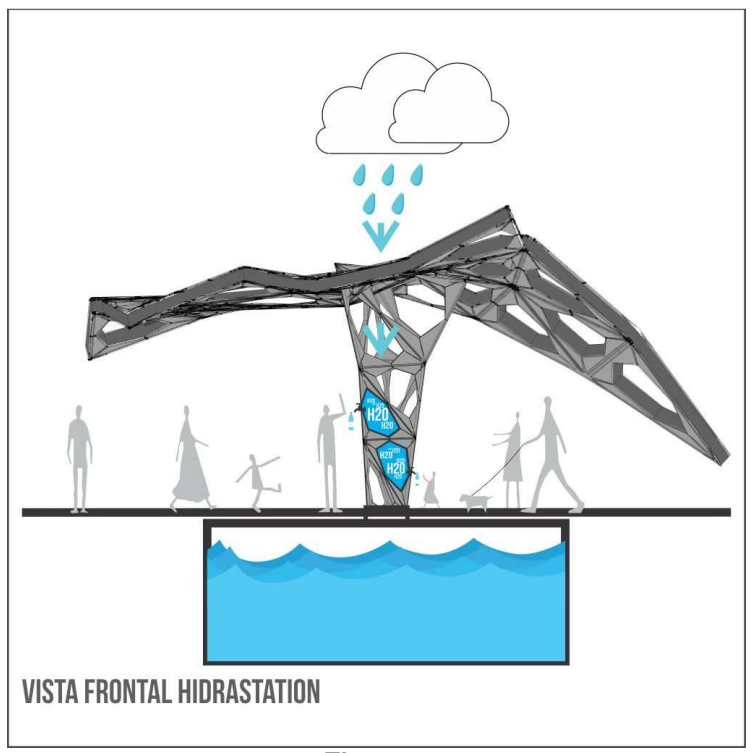

Figura 7

Por lo cual para no dejar sola a la sociedad y sus vínculos, para responder ante algunas emergencias ambientales como la carencia de agua, se propone en la Estación de Hidratación una cisterna para reciclar agua de lluvia y así mismo determinamos la provisión de los dos canales de suministros, para tener alternativas de uso ante una emergencia en algunos de ellos como lo sucedido en 2017. Esta estación se transformaría en provisora de agua para toda la ciudad contando con un sistema de control de entrega de agua, inmótico, que será usando el código del documento nacional de identidad, en caso de necesidad de racionar el agua ante una emergencia.

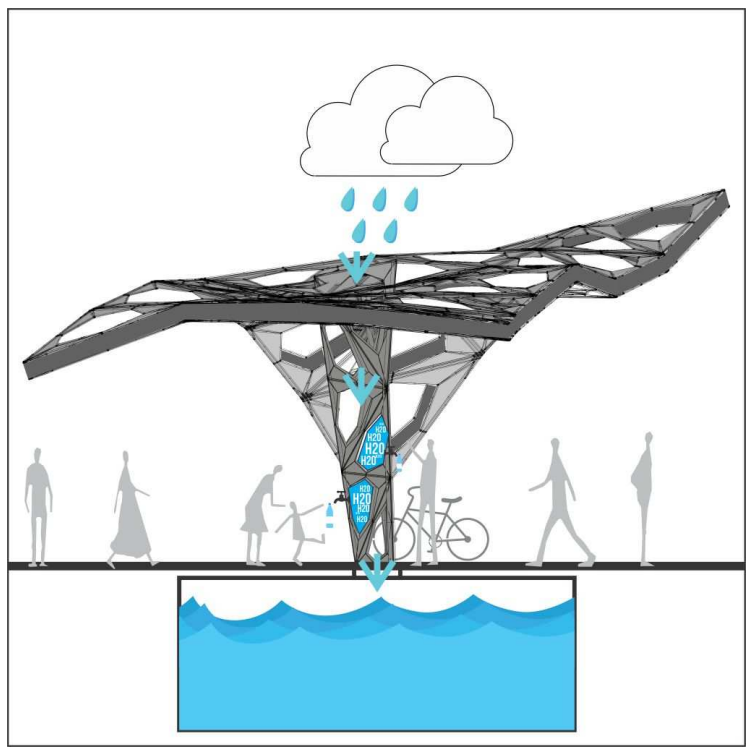

Figura 8

\section{Diseño paramétrico}

El diseño paramétrico es parte de un nuevo paradigma en la investigación proyectual ya que permite al diseñador crear sus propias herramientas, generando geometrías a partir de parámetros iniciales y un set de relaciones formales, la pérgola se despliega y en el centro recupera el agua que va a la cisterna subterránea. Diseñar paramétricamente permite trabajar tramas articuladas que en este proyecto conjuntamente con la inmótica.

Para responder a la sustentabilidad se integró el diseño paramétrico con la implementación de las células solares que posibilitan que el consumo de la estación sea provisto por energías renovables, para refrigerar la fruta, cargar celulares, etc.

Se proponen funciones automáticas de servicio, para que el usuario interactúe con ellas. En el momento de elegir si quieren servicio automático o atención de alguna apersona, los usuarios de la encuesta respondieron en un $57 \%$ que prefieren que los servicios sean automatizados.

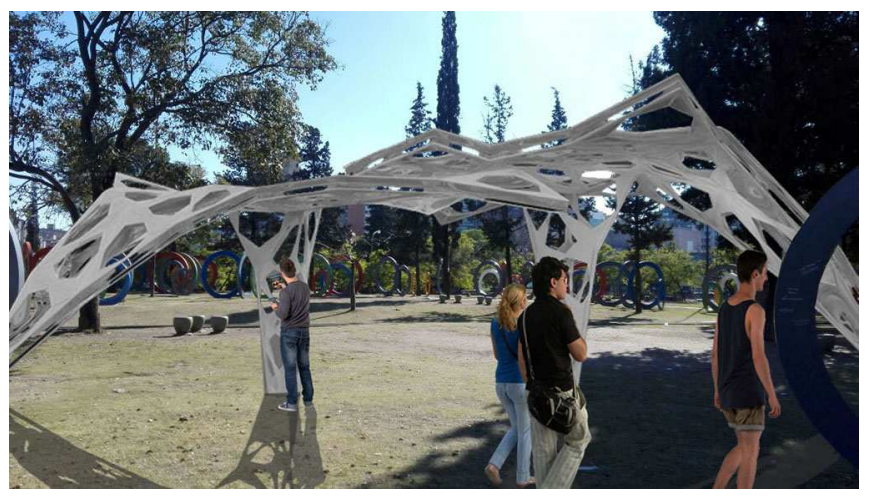

Figura 9

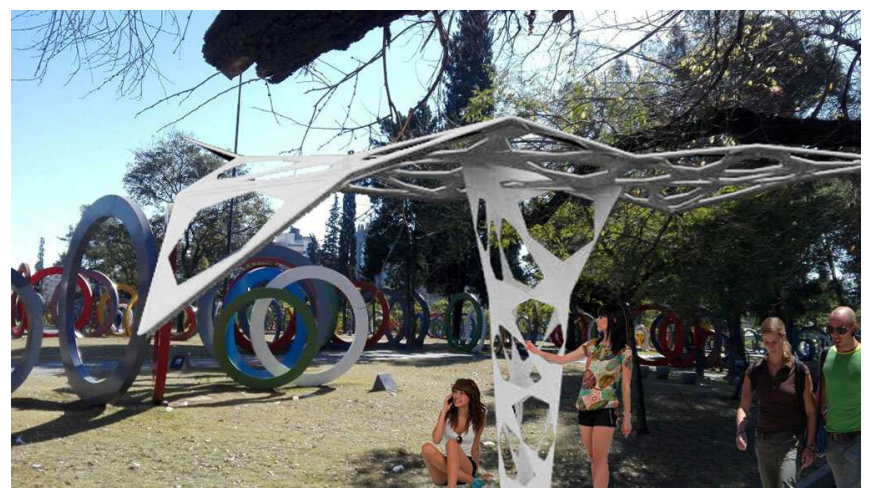

Figura 10

\section{Discusión}

¿Es posible usar la tecnología inmótica para hacer que estas microarquitectura, dialoguen con el medio y que además sean resilientes? Comprobamos con el proyecto que la tecnología 
inmótica nos permite las posibilidades de transformación, o sea podemos ser resilientes respondiendo a diferentes y a nuevos requerimientos.

\section{Importancia del proyecto}

Es un desafío para este grupo trabajar desde lo micro, equipamientos, a escala mayores y complejas como son los espacios de la ciudad. Se plantea con esta investigación el desafío de regenerar sectores, de considerar actividades y proyectar para ellas con tecnologías de vanguardia, en una sinergia sostenible e inclusiva.

La domótica y la inmótica están cada vez más relacionadas con el concepto de sustentabilidad a partir de su potencial con herramientas tecnológicas destinadas no solo a aumentar la calidad de vida en los edificios sino también y principalmente por su utilidad como recurso técnico destinado a aumentar la eficiencia energética de los sistemas construidos, el ahorro energético y por lo tanto contribuir a la sustentabilidad en el hábitat construido. Como dice el director del programa:

"Mejorar el medioambiente, es espacio público, engrandecer con el diseño que conlleva orden y que contempla la función, la morfología y lo sustentable, mediante propuestas concretas de modelos de uso urbanístico $y$ arquitectural $y$ de equipamiento urbano. Se trata de integrar servicios para la vida urbana y para la naturaleza".

El diseño de espacios que ocupen los intersticios urbanos, que responda y se adecue a su entorno, o se destaque, para los usos que la sociedad demanda es una tarea muy comprometida. Para ello es fundamental la comprensión del medio y una lectura clara detenida de su comportamiento.

\section{Agradecimientos}

Agradecemos a los asesores Ingenieros, Lucio Fernando Madussi, (lucholemm@gmail.com); Hugo Dallegre (hdallegre@gmail.com); y al Ing. Marcos Blasco, (marcos.ielect@gmail.com) de la facultad de CEFyN UNC .

\section{Referencias}

Ábalos, I. y Herrreros, J.(1997) Areas de Impunidad. Editorial Actar, Barcelona.

Adger , Adamo, (1999). Social sustainability and social resilienceof rural communities in drylands: the case of Jáchal (Argentina) in the 19th and 20th centuries. Population ResearchCenter and Department of Sociology University of Texas at Austin. Prepared for delivery at the 2003 Meeting of the Latin American Studies Association, Dallas, Texas, March27-29, 2003

Aguas cordobesas, https://www.aguascordobesas.com.ar/educacion/aulavirtual/agua-potable/potabilizacion, Recuperado junio 2017.

Augé, M.(1993), Los no lugares, Espacios de anonimato. Una antropología de la sobremodernidad. Editorial Gedisa. Barcelona.
Aureli, Pier Vittorio (2012), The Common and the Production of Architecture, Early Hypotheses, en Common Ground, A Critical Reader, Ed. David Chiperfield, Kieran Long and Shumi Bose, Marsilio Editori, Venezia,

BOHIGAS, Oriol. Reconstrucción de Barcelona. Barcelona: Edicions 62, 1985. 302 p. Trad. al cast. Reconstrucción de Barcelona. Madrid: MOPU, 1986.

Batty, M. (2005). Cities and complexity: Understanding cities through cellular automata, agent-based models, and fractals. Cambridge, MA: The MIT Press

CEDOM (2007) Asociación Española de Domótica. Cuaderno de divulgación Domótica. $2{ }^{a}$ ed. España, Barcelona: Aenor

Cabezas, C. (2014) "Claves Para Proyectar Espacios Públicos Confortables. Indicador del confort en el espacio público" 05 Jun 2014. ArchDaily. Recuperado 15 Sep 2014. http://www.plataformaarquitectura.cl/cl/02-285882/claves-paraproyectar-espacios-publicos-confortables-indicador-del-conforten-el-espacio-publico.

DELEUZE, G. 1989. El Pliegue: Leibniz y el Barroco. Barcelona, Paidós, $177 \mathrm{p}$.

Diccionario Espasa Calpe (1945), Espasa Calpe Editora ,2da Edición Argentina, Buenos Aires, México .

Deleuze, G.y Guattari, F.. Mil mesetas. Capitalismo y Esquizofrenia. Ed. De Minuit. Paris, 1980.

E2 groupe. E2 - Exploring the Urban condition. Editorial ACTAR. Paris 2003.

Fariña, F.S. (2006/2008) Predios Urbanos Intersticiales (PUInt). Validación de Herramienta de Análisis Diagnóstico Antecedente1 y Generación de Hipótesis Teóricas Fundantes de Procesos Proyectuales IDEHAB - Facultad de Arquitectura y Urbanismo SeCyT - Universidad Nacional de La Plata. Director: Arq. V. Schaposnik.

Gonzalo G. E., et al. (2007). Diseño Bioclimático de Oficinas. Pautas para San Miguel de Tucumán. Centro de Estudios Energía y Medio Ambiente, Instituto de Acondicionamiento Ambiental. Tucumán: el autor.

Hernández S. P. et al (2008) microarquitectura Equipo domotizado para auto-consulta en librería http://cumincades.scix.net/data/works/att/sigradi2008_043.conte nt.pdf.

Koolhass, R - (2000) El espacio basura, en ARQUITECTURA VIVA № 74. Madrid.

La voz del interior, http://www.lavoz.com.ar/ciudadanos/desborde-entaym-primeros-analisis-confirman-presencia-de-contaminantes recuperado junio 2017.

Maristany, Arturo (2016), http://cial.faudi.unc.edu.ar/id/investigacion/, recuperado marzo 2017

Monroy, Manuel Martin. Manual de Diseño ICARO de Calidad Ambiental en la Edificación. Departamento de Construcción Arquitectónica de la Universidad de Las Palmas de la Gran Canaria (2006).

Randriamahazo (2009), CINNERFUENTES, Exploring social resilience in Madagascar's marine protected areas. Ecology and Society 14(1): 41.[online] URL: http://www.ecologyandsociety.org/vol14/iss1 /art41/ Published here under license by theResilience Alliance.

Recuero, A. (1999) Informes de la Construcción, Vol. 50 n 459.

Schumacher, P. (2008). Digital Cities. AD Architectural Design. Parametricism. A New Global Style for Architecture and Urban Design. 79 (4) 14-23. 
Stoppani, Teresa(2012) , A Conversation of Many, en Common Ground, A Critical Reader, Ed. David Chiperfield, Kieran Long and Shumi Bose, Marsilio Editori, Venezia,

Monroy, Manuel Martin. Manual de Diseño ICARO de Calidad Ambiental en la Edificación. Departamento de Construcción Arquitectónica. Universidad de Las Palmas de la Gran Canaria ,2006.

KCOMT, Natalie Ché et al, (2010), An intelligent domotics system to automate user Actions, davy.preuveneers.be/publications/isami10b.pdf, recuperado enero 2012

Primer congreso de edificios inteligentes. (2013). España, 23 y 24 octubre. [Consulta octubre 2013]. Disponible en: http://www.congreso-edificiosinteligentes.es/
ROMERO MORALES C. et al.(2006). Domótica e Inmótica: viviendas y edificios inteligentes. $2^{\mathrm{a}}$ ed. Madrid: Ra-Ma.

SCHUMACHER, P. 2008. Digital Cities. AD Architectural Design. Parametricism. A New Global Style for Architecture and Urban Design. 79 (4) 14-23 